\title{
The SAGE handbook of transport studies
}

\author{
M. Wilde \\ Institut für Humangeographie, Goethe-Universität Frankfurt am Main, Frankfurt am Main, Germany \\ Correspondence to: M. Wilde (wilde@geo.uni-frankfurt.de)
}

Rodrigue, J.-P., Notteboom, T., and Shaw, J. (Ed.): The SAGE handbook of transport studies, Sage, London, 448 pp., ISBN-13: 978-1849207898, €112, 2013.

Der Verkehrsgeographie sagt man zuweilen nach, sie schaffe es nicht, ihren szientistischen Ursprung hinter sich zu lassen und an die aktuellen Entwicklungen der Humangeographie anzuschließen. Gewiss kann einem Teil der geographischen Verkehrsforschung eine Abstinenz gegenüber theoretischer Vielfalt attestiert werden, aber in den letzten Jahren entwickelte sich das Fachgebiet geradezu rasant. Die Verkehrsforschung durchbricht die Grenzen von Disziplinen, nutzt deren Theorienvielfalt für ihr Fachgebiet und entwirft eine eigene Sichtweise auf die Welt. Dass sich die Kritik an der geographischen Verkehrsforschung längst überholt hat, davon kann man sich in zahlreichen Lehrbüchern überzeugen.

Seit Neuerem gehört „The SAGE Handbook of Transport Studies“ dazu. Dieser Sammelband wurde von den Verkehrsgeographen Jean-Paul Rodrigue und Jon Shaw sowie dem Verkehrsökonomen Theo Notteboom herausgegeben. Das Handbuch trägt eine geographische Handschrift, es geht aber weit über die Perspektive der Verkehrsgeographie hinaus. Insgesamt 25 Kapitel wurden von 37 Autoren und Autorinnen geschrieben - von der Geographie, über die Wirtschaftsund Ingenieurswissenschaften bis hin zu Politik und Gesundheitswesen versammelt das Buch die Hautevolee der Verkehrsforschung. Die Herausgeber stellen die Beiträge so zusammen, dass die Leser und Leserinnen einen möglichst umfangreichen Einblick in die aktuellen wie auch altbewährten Themen der Verkehrsforschung erhalten.

Das Handbuch besteht aus sechs thematischen Schwerpunkten. So setzen sich die ersten beiden Teile, Transport and the global world und Transport in regions and localities, mit der Ausdehnung, Beschaffenheit und Funktion von Verkehrssystemen auf verschiedenen räumlichen Maßstabsebenen auseinander. Diese zwei Abschnitte spiegeln noch die klassische Verkehrsgeographie als eine Disziplin wider, die sich vor allem mit der Beschreibung des Zwecks, der Anord- nung und Organisation von Infrastrukturen begnügt. Allerdings geben die verbleibenden Abschnitte schnell zu erkennen, dass der klassische Einstieg bewusst gewählt wurde. Er schafft unentbehrliche Grundlagen, die es den Lesern ermöglichen, die weiteren Ausführungen in räumliche Zusammenhänge einzuordnen. Denn die folgenden Beiträge gehen viel bestimmter der Frage nach, wie Mobilität und Verkehr sich als Glassplitter in das Mosaik des gesellschaftlichen Zusammenlebens einfügen. Die Abschnitte Transport, economy and society und Transport policy befassen sich mit den gesellschaftsrelevanten Implikationen von Mobilität und Verkehr, ordnen sie in wirtschaftliche und politische Zusammenhänge ein. Daran schließt sich Transport Networks und Models an. Dieser Abschnitt greift die eher technische, von Ingenieuren vertretene Verkehrsforschung auf, stellt Modelle und Prognosemethoden vor, auf denen die heutige Infrastrukturplanung fast ausnahmslos basiert. Umwelt und Nachhaltigkeit sind die Themen des letzten Abschnittes, Transport and the environment, der neben Ausführungen zum Energieverbrauch und Klimawandel auch einen Beitrag enthält, der auf eine an den Prinzipien der Nachhaltigkeit orientiere Logistik eingeht (Green Supply Chain Management). Das ist insofern bemerkenswert, als dass in Betrachtungen zur Nachhaltigkeit der Wirtschaftsverkehr gewöhnlich weit unterrepräsentiert ist.

„The SAGE Handbook of Transport Studies“ nimmt den Leser mit auf eine Reise durch die Verkehrsforschung. Die Herausgeber legen ein Werk vor, das die wesentlichen Strömungen für jeden verständlich aufzeigt, das sich nicht in Details verliert, aber dennoch umfangreich informiert. Zahlreiche Karten, Tabellen und Schaubilder begleiten den Text und erläutern kompliziertere Sachverhalte. Die Abbildungen sind von außergewöhnlicher Schärfe, ihnen ist anzusehen, dass sie von Grafikern und Kartographen erstellt wurden, die ihre Kunst verstehen. Allein ein allzu enger Fokus auf die westlichen Industrienationen trübt den guten Gesamteindruck. Im Handbuch dominiert der Blick von Wissenschaftlern und Wissenschaftlerinnen aus Nordamerika und Westeuropa, allenfalls von einer Außenperspektive schauen sie 
auf die Verhältnisse in den Schwellen- oder Entwicklungsländern.

Von „The SAGE Handbook of Transport Studies“ darf man keine komplett neuen Erkenntnisse erwarten. Der Wert liegt in der Zusammenschau, mit der es den Autoren und Autorinnen gelingt, den Lesern die Fülle und Vielfältigkeit der Verkehrsforschung näherzubringen. Lehrende und Forschende der Geographie, der Raumplanung, der Wirtschaftswissenschaft und jeder anderen mit Mobilität und Verkehr befassten Disziplin finden klar strukturierte, sorgfältig recherchierte Überblicksartikel. Studierenden kann das Handbuch als Wegweiser dienen, der einen Pfad durch das geradezu undurchdringliche Dickicht heutiger Verkehrsforschung aufzeigt. 